\title{
ELECTRIC POLARIZATION OF ONSAGER FLUIDS. 1. DIPOLE POLARIZATION 2. BINARY SOLUTIONS OF ORGANIC COMPOUNDS
}

\author{
V.V. Prezhdo ${ }^{b}$, S.A. TYURIN ${ }^{a}$, O.V. Prezhdo ${ }^{c}$, G.V. TARAsova ${ }^{a}$, \\ O.N. AKulova ${ }^{a}$ AND T.N. KURSKAYA ${ }^{a}$ \\ ${ }^{a}$ Ukrainian Scientific Center for Water Control, Bakunina 6, Kharkov 310001, Ukraine \\ ${ }^{b}$ Institute of Chemistry, Pedagogical University, Oleska 48, 45-052 Opole, Poland \\ ${ }^{c}$ Kharkov University, pl. Nezalezhnosti 4, Kharkov 310077, Ukraine
}

(Received May 14, 1993; in final form Oclober 19, 1993)

Set forth in the previous report [1], the continual theory of dipole polarization of molecular fluids, which suggests substitution of the rigid Onsager sphere for the polarizable body and takes into consideration electric induction of this body and its nearest surroundings, is extended in this paper to binary solutions with diverse polarities. Dipole moments of free molecules of dissolved substances were calculated from experimental data of dielectrometry of a large number of extremely diluted organic substances and are close to the corresponding gaseous phase values. On the ground of solution polarization measurements the criterion of physical consistency of various models of the condensed medium internal field is proposed.

PACS numbers: $35.20 . \mathrm{My}, 31.70 . \mathrm{Dk}$

\section{Introduction}

Determinations of electric properties of free molecules of substances which do not exist in gaseous phase or cannot be transformed into it (when such a transformation may cause destruction of molecule or changes in molecular conformations and functional group energies) are carried on by studying corresponding properties in solutions. Necessity to consider dispersive, inductive and dipole-dipole interactions of molecules of a solute, both with each other and with molecules of a solvent, establishes salient features of this approach. The theories of condensed medium polarization, widely developed in recent years, are based on statistic and molecular as well as computer simulation techniques. They usually show theoretical heuristic results and include molecular medium parameters that are difficult to be obtained from experiments. Whereas continual theories of dipole polarization 
which assume the absence of intermolecular interaction can model solvent effects with various degree of certainty applying effective characteristics of exerted fields and molecular moments. Giving preference to the Onsager theory and its modifications we have performed a research with the goal of both to examine calculated values of dipole moments of substances dissolved in non-polar and polar matrices and to select a reliability assessment criterion for model description of internal field in condensed media. The objects of our investigation are binary, extremely diluted solutions with a negligible interaction between dissolved molecules.

\section{The model of solution polarization. Determination of dipole moments.}

As a beginning some molecular and macroscopic characteristics of a binary solution and its components: $x_{i}=N_{i}\left(\sum N_{i}\right)^{-1}, \sum x_{i}=1$ - mole fractions of solution components, $N_{i}$ - a number of molecules per unit volume, $\rho_{i}$ and $M_{i}$ - densities and molecular weights, $\varepsilon_{\infty i}$ and $\varepsilon_{i}$ - high and low frequency dielectric permittivities, $\mu_{i}^{0}$ - dipole moments of free molecules; the values of dipole moments $\mu$ and auxiliary parameters are referred to the solutions while indexes $i=1$ or 2 denote a solvent and a solute, respectively. Staying within the approximation of absence of intermolecular coupling, we can write the Maxwell equation which binds the electric field affecting a dielectric medium and the polarizational response of the medium in the following way:

$$
(\varepsilon-1) \rho^{-1}=4 \pi N_{\mathrm{A}}\left(P_{\mathrm{m} 1}\left(1-x_{2}\right) M_{1}^{-1}+P_{\mathrm{m} 2} x_{2} M_{2}^{-1}\right),
$$

where additive molecular polarizations are calculated according to the optative ideas on dipole polarization of liquids. In particular, the modified Onsager theory presented in our previous report [1] defines $P_{\mathrm{m} i}(i=1$ or 2$)$ as

$$
P_{\mathrm{m} i}=g\left[\frac{1+1.5 \alpha_{i} t}{\left(1-\alpha_{i} t\right)^{3}} \frac{\left(\mu_{i}^{0}\right)^{2}}{3 k T}+\frac{\alpha_{i}}{1-\alpha_{i} t}\right]=g P_{\mathrm{m} i}^{*}
$$

( $N_{\mathrm{A}}$ and $k$ are the Avogadro and Boltzmann constants, $g$ and $t$ - cavity and reactive field factors at the absolute temperature $T, \alpha_{1}$ are dynamic polarizabilities of the molecules). Assumption is made that the Onsager fields are characterized by the parameters of a solution in the aggregate

$$
G_{i}^{*}=g_{i}^{*} E=g E\left(1-\alpha_{i} t\right)^{-1} \text { and } R_{i}^{*}=t m_{i}\left(1-\alpha_{i} t\right)^{-1} .
$$

Employing the most preferable scheme of determination of any extensive property of a substance dissolved in a solution - extrapolating its concentration to zero $\left(x_{2} \rightarrow 0\right)$, by differentiating both sides of Eq. (1) under condition that $\rho\left(x_{2}\right), \varepsilon\left(x_{2}\right)$ and $n\left(x_{2}\right)$ are linear functions we obtain

$$
\begin{aligned}
& \frac{\varepsilon_{1}-1}{\rho_{1} g_{1}}\left(1-\beta-\frac{1}{g_{2}} \frac{\mathrm{d} g}{\mathrm{~d} x_{2}}+\frac{\delta \varepsilon_{1}}{\varepsilon_{1}-1}\right)_{x_{2}=0} \\
& \quad=4 \pi N_{\mathrm{A}}\left(\frac{P_{\mathrm{m} 2}^{*}}{M_{2}}+\frac{1-x_{2}}{M_{1}} \frac{\mathrm{d}}{\mathrm{d} x_{2}} P_{\mathrm{m} 1}^{*}\right)_{x_{2}=0}, \\
& \rho=\rho_{1}\left(1+\beta x_{2}\right), \quad \varepsilon=\varepsilon_{1}\left(1+\delta x_{2}\right), \quad n=n_{1}\left(1+\gamma x_{2}\right) .
\end{aligned}
$$


All derivatives in Eq. (3) are taken at $x_{2}$ equal to zero and

$$
4 \pi N_{\mathrm{A}} P_{m 1}^{*} M_{1}^{-1}=\left(\varepsilon_{1}-1\right)\left(g_{1} \rho_{1}\right)^{-1} \text {. }
$$

When we also consider volume fractions of solution components

$$
\begin{aligned}
& A_{1}=M_{1} \rho_{2}\left(1-x_{2}\right)\left[M_{1} \rho_{2}\left(1-x_{2}\right)+M_{2} \rho_{1} x_{2}\right]^{-1}, \\
& A_{2}=M_{2} \rho_{1} x_{2}\left[M_{1} \rho_{2}\left(1-x_{2}\right)+M_{2} \rho_{1} x_{2}\right]^{-1}, \quad A_{1}+A_{2}=1,
\end{aligned}
$$

then the calculation with Eq. (3) yields the final relationships which determine dipole moments of free molecules of a substance $\left(\mu_{2}^{0}\right)$ dissolved in the

a) non-polar $\left(\mu_{1}^{0}=0\right)$ solvent

$$
\begin{aligned}
& \frac{4 \pi N_{\mathrm{A}}}{3 k T M_{2}} \frac{1+1.5 \alpha_{2} t_{1}}{\left(1-\alpha_{2} t_{1}\right)^{3}}\left(\mu_{2}^{0}\right)^{2}= \\
& \frac{\varepsilon_{1}-1}{\rho_{1} g_{1}}\left[1-\beta+\frac{\delta \varepsilon_{1}}{\varepsilon_{1}-1}+\frac{M_{2} \rho_{1}}{M_{1} \rho_{2}}-\frac{\mathrm{d} \ln g}{\mathrm{~d} x_{2}}-\frac{1}{1-\alpha_{i} t_{i}} \frac{\mathrm{d}}{\mathrm{d} x_{2}}\left(\alpha_{1} t\right)\right]_{x_{2}=0}, \\
& \text { b) polar }\left(\mu_{1}^{0} \neq 0\right) \text { solvent } \\
& \frac{4 \pi N}{3 k T M_{2}} \frac{1+1.5 \alpha_{2} t}{\left(1-\alpha_{2} t_{1}\right)^{3}}\left(\mu_{2}^{0}\right)^{2}= \\
& \frac{\varepsilon_{1}-1}{\rho_{1} g_{1}}\left[1-\beta+\frac{\delta \varepsilon_{1}}{\varepsilon_{1}-1}-\frac{\mathrm{d} \ln g}{\mathrm{~d} x_{2}}-\frac{3\left(1.5+\alpha_{1} t_{1}\right)}{\left(1-\alpha_{1} t_{1}\right)\left(1+1.5 \alpha_{1} t_{1}\right)} \frac{\mathrm{d}\left(\alpha_{1} t\right)}{\cdot \mathrm{d} x_{2}}\right]_{x_{2}=0} \\
& +\frac{3\left(n_{1}^{2}-1\right)}{\rho_{1}\left(n_{1}^{2}+2\right)} \frac{1}{1-\alpha_{1} t_{1}}\left[\frac{3\left(1.5+\alpha_{1} t_{1}\right)}{\left(1-\alpha_{1} t_{1}\right)\left(1+1.5 \alpha_{1} t_{1}\right)} \frac{\mathrm{d}\left(\alpha_{1} t\right)}{\mathrm{d} x_{2}}+\frac{M_{2} \rho_{1}}{M_{1} \rho_{2}}\right]_{x_{2}=0} .
\end{aligned}
$$

The factors $g, t$ and their derivatives with respect to the dissolved substance concentration $x_{2}$ are specified by the manner of description of internal field in condensed dielectrics. The Onsager body radii $a_{i}$ in the term which defines $\alpha_{i}$ and $t_{i}$ are taken in accordance with [2] in the form $a_{i}^{3}=(0.86 \mp 0.03) r^{3}$, where $r$ are the Van der Waals radii of molecules of solution components.

\section{Experimental data on dielectrometry of extremely diluted binary solutions}

To test the above outlined method of estimation of free dipole moments $\left(\mu_{2}^{0}\right)$ of molecules in solutions and to compare the results with other approaches, we performed electrometric studies of a great number of binary solutions (with $x_{2}$ less than 0.02) and their polar and non-polar components.

We used standard equipment with the following measurement errors: density error $(\Delta \rho)$ - less than $0.05 \%$, dielectric constant $(\varepsilon)$ - within $0.03 \%$, refraction index $(n)$ - within $0.02 \%$. The data obtained for solutions in non-polar solvents are presented in Table, and the results for polar solvents are depicted in Figs. 1 and 2. Pure organic compounds with tabulated gaseous phase values of dipole moments [3] were selected as reference components of solutions. Thus, we chose the following solvents for the first series of binary solutions (see Table): $n$-hexane $\mathrm{CH}_{3}\left(\mathrm{CH}_{2}\right)_{4} \mathrm{CH}_{3}(1)$, tetrachloromethane $\mathrm{CCl}_{4}(2)$, benzene $\mathrm{C}_{6} \mathrm{H}_{6}(3)$, 1,4-dioxane 
TABLE

Experimental and calculated characteristics of diluted solutions of polar substances (6-8) in non-polar solvents (1-5).

\begin{tabular}{l|r|r|r|r|r|r|c}
\hline \hline \multirow{2}{*}{$\begin{array}{c}\text { Sol- } \\
\text { vent }\end{array}$} & \multicolumn{7}{c}{ Dissolved substance } \\
\cline { 3 - 8 } & & $\gamma$ & $\delta$ & \multicolumn{4}{|c}{$\Delta \mu_{2}^{0}$} \\
\cline { 4 - 7 } & & & $A$ & $B$ & $C$ & $D$ \\
\hline 1 & -0.04661 & -0.13989 & 14.20252 & 0.0722 & 0.0515 & 0.0303 & 0.0673 \\
3 & 0.58442 & 0.19383 & 22.35439 & 0.0614 & 0.0428 & 0.0198 & 0.0584 \\
4 & -0.36157 & 0.19267 & 9.30621 & 0.0537 & 0.0349 & 0.0152 & 0.0531 \\
5 & 0.89680 & 0.36646 & 9.57097 & 0.0483 & 0.0304 & 0.0097 & 0.0472 \\
\hline \multicolumn{7}{c}{ Cyclopentanone } \\
\hline 1 & -0.38001 & -0.51024 & 9.37974 & 0.0596 & 0.0426 & 0.0198 & 0.0554 \\
2 & -0.24123 & -0.15535 & 9.56405 & 0.0535 & 0.0351 & 0.0151 & 0.0509 \\
3 & -0.17408 & -0.31075 & 11.41150 & 0.0502 & 0.0289 & 0.0099 & 0.0467 \\
4 & 0.24503 & -0.37704 & 5.86291 & 0.0461 & 0.0250 & 0.0056 & 0.0415 \\
5 & 0.18915 & -0.06194 & 4.18215 & 0.0410 & 0.0200 & 0.0049 & 0.0366 \\
\hline \multicolumn{7}{c}{$t$-Butyl chloride } \\
\hline 1 & -0.32330 & -0.14368 & 3.16824 & 0.0408 & 0.0199 & 0.0050 & 0.0458 \\
2 & -0.17448 & -0.18399 & 3.07638 & 0.0348 & 0.0153 & 0.0054 & 0.0408 \\
3 & -0.02989 & -0.06926 & 2.61217 & 0.0286 & 0.0105 & 0.0049 & 0.0361 \\
4 & -0.66320 & -0.04310 & 2.63564 & 0.0251 & 0.0054 & 0.0033 & 0.0328 \\
5 & 0.29011 & 0.01604 & 2.14162 & 0.0217 & 0.0048 & 0.0019 & 0.0244
\end{tabular}

$\mathrm{C}_{4} \mathrm{H}_{8} \mathrm{O}_{2}$ (4) and carbon disulphide $\mathrm{CS}_{2}$ (5) as well as the following solutes: nitrobenzene $\mathrm{C}_{6} \mathrm{H}_{5} \mathrm{NO}_{2}(6)$, cyclopentanone $\mathrm{C}_{5} \mathrm{H}_{8} \mathrm{O}(7)$ and 2-methyl-2-chloro-propane $\left(\mathrm{CH}_{3}\right)_{3} \mathrm{CCl}(8)$. The second series of solvents were polar organic compounds, viz. halogenomethanes, amines, ethers, alcohols, as well as aromatic compounds like alkyl and halogeno derivatives of benzene, while the solutes were: benzonitrile $\mathrm{C}_{6} \mathrm{H}_{5} \mathrm{CN}(9)$, nitrobenzene (6) and salicyl aldehyde $\mathrm{C}_{6} \mathrm{H}_{5}(\mathrm{OH}) \mathrm{CHO}(10)$. The results are presented in Fig. 1 and Fig. 2.

The values of dipole moments in solutions were calculated within the framework of the continual theory of polarization of liquids and compared with the data obtained from measurements performed in gaseous phase (as $\Delta \mu$ ). The differences $\Delta \mu$ were plotted against solvent specific polarizabilities $\left(P_{1}^{*}\right)$ (Fig. 1) and dipole moments of the solvents (Fig. 2). For each compound $(6,9$ and 10) a series of curves has been obtained due to different methods used for calculations. The curve $A$ comes from the Onsager model of internal field [4] and the $B$ series from its modification $[5,6]$ which includes the local dielectric permittivity $\varepsilon(r)$. Calculations according to Eq. (4) yielded curves signed $C$, whereas the $D$ and $E$ series 

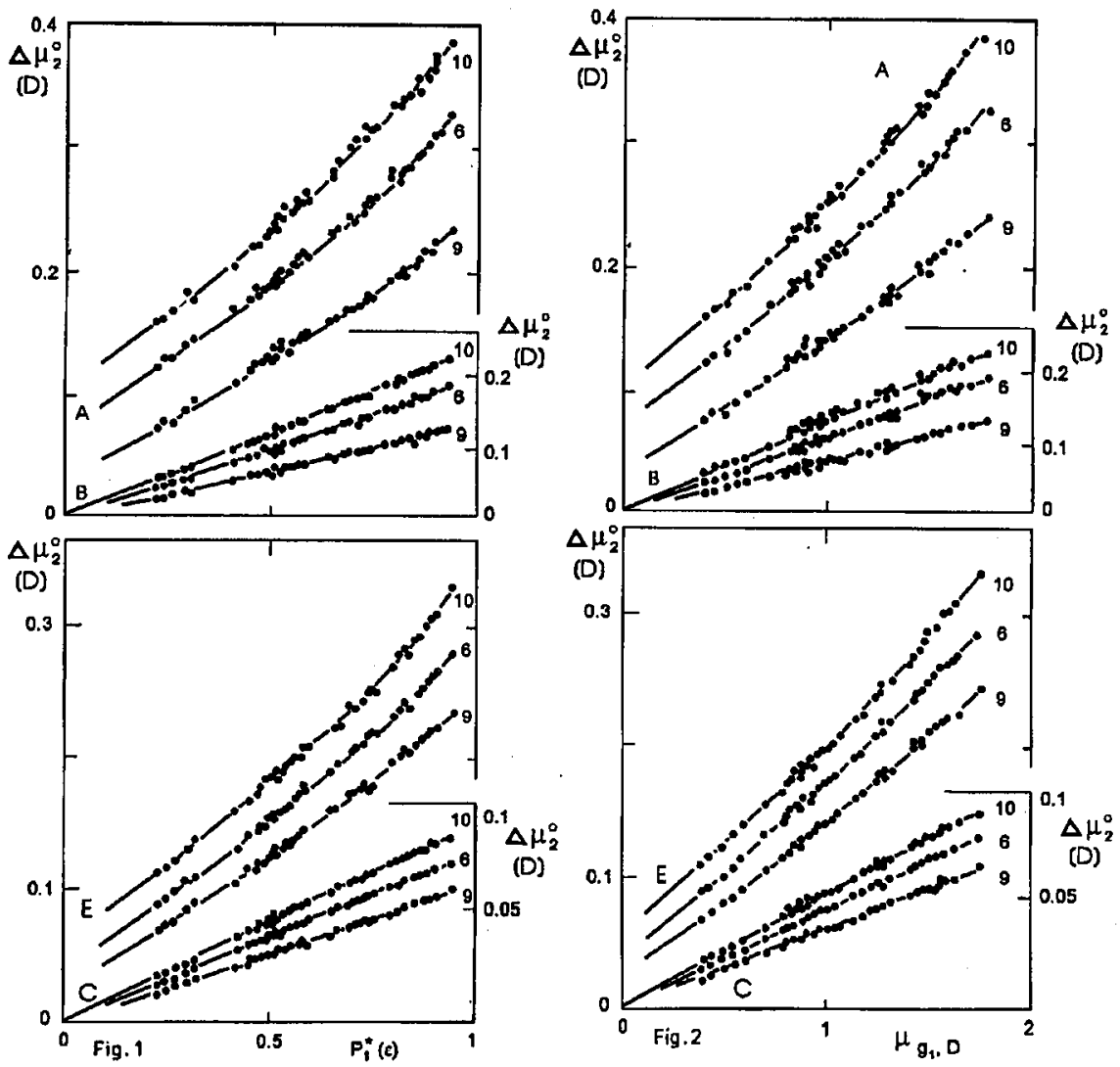

Fig. 1. Differences $\Delta \mu_{2}^{0}$ between dipole moments estimated in solutions and the values calculated from measurements in gaseous phase versus solvent specific polarizability $P_{1}^{*}(\varepsilon)$

Fig. 2 The difference $\Delta \mu_{2}^{0}$ versus dipole moments of solvents, estimated in gaseous phase $\mu_{\mathrm{g} 1}$.

were obtained by extrapolation methods [7, 8], traditionally used in physicochemical analyses of solutions in non-polar and polar solvents, respectively.

\section{Discussion of the results and conclusions}

Extremely diluted solution of polar molecules in a non-polar solvent within the limits of the Onsager model can be considered in a certain sense as a gas of polar particles. However, when studying the effects of dipole polarization of solutions one should expect distinct manifestations of solute-solvent intermolecular interactions which causes the increase in dynamic polarizability of molecules of the latter. The data in Table confirm this assumption: the greatest values of deviations in dipole moments of solute molecules $\left(\Delta \mu^{0}\right)$ are detected in $n$-hexane $\left(1, \alpha=11.85 \AA^{3}\right)$, while the smallest - in carbon disulphide $\left(5, \alpha=8.52 \AA^{3}\right)$. Solvent polarity has 
analogous influence on the deviations $\Delta \mu^{0}$ of nitrobenzene $\left(6, \mu^{0}=4.23 \mathrm{D}\right)$ and exceeds $\Delta \mu^{0}$ of 2-methyl-2-chloro-propane $\left(8, \mu^{0}=2.11 \mathrm{D}\right)$ in all solvents used.

Extremely diluted binary solutions whose both components are polar differ from those discussed above mainly in the nature and magnitude of the intermolecular interactions.

This fact reveals itself in similar changes of dipole moment deviations with the rise in polarizabilities and dipole moments of molecules of solvents (Fig. 1). Again, in common with the first series of solvents, the larger solute dipoles $\left(\mu^{0}\right)$, the greater the errors: $\Delta \mu$ of benzonitrile (I) $\left(\mu^{0}=4.39 \mathrm{D}\right)$ exceeds $\Delta \mu$ of salicyl aldehyde (10) $\left(\mu^{0}=3.10 \mathrm{D}\right)$ in all solvents. And finally it is necessary to stress the salient manifestation of intermolecular interactions which result in a systematic overrun in dipole moment deviations in polar solutions over those in non-polar ones. For instance, the differences of calculated dipole moments of nitrobenzene (6) in benzene (3) (Table) which has molar polarizability $\left(P_{1}^{*}\right)$ equal to 0.299 , are approximately 40 per cent of those in a polar solvent with the same polarizability (Fig. 1).

It is well known that one of the most substantial drawbacks of the continual theories of electric polarization of condensed molecular media is the assumption of absence of intermolecular interactions. The Onsager theory is not an exception. However, polarizational response of the Onsager cavity in terms of reactive field introduced into the structure of internal field by Onsager himself can be considered, though not in an explicit form, as the first approximation to this interactions. This fact favourably distinguishes the Onsager theory from others. In this connection further development of the concepts of reactive field and molecular distribution locality is thought as a purposeful search for a physically adequate model of dipole polarization of condensed phases, in particular, binary solutions.

The results of the application of the Onsager internal field models, mentioned above, to calculations of dipole moments of free molecules of substances dissolved in non-polar and polar solvents are presented in Table and in Figs. 1 and 2 , respectively. Independently from polarities of components of binary solutions investigated the smallest computed dipole deviations $\left(\Delta \mu^{0}, C\right)$ are observed in the calculations by Eq. (4) which are based on the substitution of the rigid Onsager sphere with a polarizing body and employ dielectric permittivity function related to microcharacteristics of a substance by the Fresnel integrals [6]. Another modification of the internal field theory which replaces local permittivity of Heaviside's type with that described in [5] leads to somewhat worse results as evidenced by the data on $\Delta \mu(B)$ (Fig. 1 and Fig. 2). However, with the decrease in both $\mu_{\mathrm{g} 1}$, $\mu_{g 2}$ and dynamic polarizabilities of solution components value of $\Delta \mu(B)$ is equal with $\Delta \mu(C)$. In our opinion, with diminishing contribution in dipole polarization of a liquid not only reactive fields of electric dipole moments of molecules, but also reactive fields induced in the Onsager body of multipoles decrease. Although we cannot state confidently, because of lack of reliable experiments on solutions with non-polar solvents having small polarizabilities $\left(P_{1}^{*}<0.2\right)$, that dipole moment deviations of solutes in polar and non-polar solvents coincide when solvent polarizability tends to zero $\left(P_{1}^{*} \rightarrow 0\right)$, such a tendency is rather distinct. The greatest deviation of dipole moments $\mu_{2}^{0}$ of substances dissolved in any of the solvents used, 
from their gaseous phase values, are observed in calculations within the limits of the classical Onsager theory. And lastly, gradual decrease in the gradients of the functions $\Delta \mu\left(\mu_{1}^{0}, P_{1}^{*}\right)$ (Fig. 1 and Fig. 2) is an additional evidence in favour of a credible relationship of the above-mentioned modifications of the Onsager approach with intermolecular interactions, in particular of the solvent-solute type.

It is possible to presume the following reasons for the aforenamed trends of dielectrometry of binary solutions. We can look at the extrapolation of the data (Fig. 1 and Fig. 2) to the axis of abscissae $(\Delta \mu=0)$ as at an approximation to the aforesaid analogy between the gaseous state of a substance and its extremely diluted solution, i.e. to the "perfectly inert" solvent. The latter should be probably considered as a hypothetical compound with non-polar and non-polarizable molecules. The best results of such an extrapolation are exhibited by the dipole moment calculations performed within the modified Onsager theories $(B, C)$ which include both, the bulk effects of polarization of the Onsager body and reactive fields induced in the body, and local distribution of electric induction of the nearest to fixed polar molecule continuous surroundings: $P_{1}^{*}, \mu_{\mathrm{g}} \approx 0$. The similar processing of the deviations $\left(\Delta \mu^{0}\right)$ obtained according to the Onsager theory $(A)$ and extrapolation expressions $(E)$ [7], yields physically unreal parameters of the hypothetical solvent: $P_{1}^{*}, \mu_{\mathrm{g}}<0$. This fact in combination with the preceding inferences can be thought of as a reliable criterion of physical consistency of both basic premises of model descriptions of dielectric media and methods of determination of dipole moments of free molecules of solutes.

Finally, the analysis of the results of investigation of extremely diluted binary solutions presented here, indicates that development of the concepts of reactive field and local dielectric permittivity in the context of continual theories of polarization of molecular liquids enables us to estimate dipole moments of free molecules as close as possible to their gas phase values.

\section{References}

[1] V.V. Prezhdo, S.A. Tyurin, O.V. Prezhdo, G.V. Tarasova, O.N. Akulova, T.N. Kurskaya, Acta. Phys. Pol. A 84, 253 (1993).

[2] V.A. Gorodysskiy, N.G. Bakhshiev, L.F. Kardashina, Spektrokhimiya Vnutrii Mezhmolekulyarnykh Vzaimodeystviy (USSR) 1, 63 (1975).

[3] A.L. McLelland, Tables of Experimental Dipole Moments, W.H. Freeman and Co., San Francisco, Vol. 1, 1963, Vol. 2, 1973, Vol. 3, 1989.

[4] L. Onsager, J. Am. Chem. Soc. 58, 1486 (1936).

[5] H. Block, S.M. Walker, Them. Phys. Lett. 19, 363 (1973).

[6] S.A. Turin, M.V. Khashchina, Zh. Fiz. Khim. 61, 114 (1987).

[7] M.F. Vuks, Zh. Fiz. Khim. 52, 134 (1978).

[8] S.B. Bulgarevich, A.A. Polunin, O.A. Obispo, V.A. Koran, Teor. Eksp. Khim. 14, 842 (1977). 